PROCEEDINGS OF THE

AMERICAN MATHEMATICAL SOCIETY

Volume 134, Number 10, October 2006, Pages 3049-3056

S 0002-9939(06)08313-4

Article electronically published on April 13, 2006

\title{
ASYMPTOTICALLY CYLINDRICAL RICCI-FLAT MANIFOLDS
}

\author{
SEMA SALUR \\ (Communicated by Richard A. Wentworth)
}

\begin{abstract}
Asymptotically cylindrical Ricci-flat manifolds play a key role in constructing Topological Quantum Field Theories. It is particularly important to understand their behavior at the cylindrical ends and the natural restrictions on the geometry. In this paper we show that an orientable, connected, asymptotically cylindrical manifold $(M, g)$ with Ricci-flat metric $g$ can have at most two cylindrical ends. In the case where there are two such cylindrical ends, then there is reduction in the holonomy group $\operatorname{Hol}(g)$ and $(M, g)$ is a cylinder.
\end{abstract}

\section{INTRODUCTION}

Let $\left(M^{n}, g\right)$ be a connected asymptotically cylindrical manifold with Ricci-flat metric $g$. Examples of such manifolds are asymptotically cylindrical Riemannian manifolds whose holonomy groups $\operatorname{Hol}(g)$ are subgroups of $S U(m)$, for $n=2 m$, and subgroups of the exceptional Lie group $G_{2}$, for $n=7$. Examples of asymptotically cylindrical Riemannian 6-manifolds with holonomy $S U(3)$ were first constructed by Tian and Yau [12, [13] and Kovalev [5]. In his paper, [5], Kovalev also showed that the Riemannian product of these 6 -manifolds with a circle produced asymptotically cylindrical Riemannian 7-manifolds with holonomy $S U(3) \subset G_{2}$. These are the first examples of asymptotically cylindrical $G_{2}$ manifolds.

Other examples of noncompact Ricci-flat manifolds $\left(M^{n}, g\right)$ are hyperkähler manifolds, manifolds with holonomy group $\operatorname{Hol}(g) \subset S p(m)$, for $n=4 m$, and $\operatorname{Spin}(7)$ manifolds with holonomy group $\operatorname{Hol}(g) \subset \operatorname{Spin}(7)$, for $n=8$.

Asymptotically cylindrical Calabi-Yau and $G_{2}$ manifolds are the main objects of the Topological Quantum Field Theories, as is explained in [6]. In order to construct consistent TQFT's, it is essential to understand the geometric restrictions at the cylindrical ends of a given orientable, connected, noncompact Ricci-flat manifold with some small decay rate. A very natural and fundamental question is whether it is possible for these manifolds to have multiple cylindrical ends.

In this paper we prove the following theorem.

Theorem 1.1. Let $(M, g)$ be an orientable, connected, asymptotically cylindrical Riemannian manifold with Ricci-flat metric $g$ and with $l$ cylindrical ends. Then

Received by the editors November 17, 2004 and, in revised form, April 7, 2005 and April 26, 2005.

2000 Mathematics Subject Classification. Primary 53C15, 53C21; Secondary 58J05.

Key words and phrases. Differential geometry, global analysis, analysis on manifolds.

This research was supported in part by AWM-NSF Mentoring grant.

(C)2006 American Mathematical Society Reverts to public domain 28 years from publication 
$l \leq 2$, and in the case when $l=2$ there is reduction in the holonomy group $\operatorname{Hol}(g)$ and $(M, g)$ is a cylinder.

Remark 1.2. The proof of this theorem also follows from the Cheeger-Gromoll splitting theorem [2. The key ingredients of the splitting theorem are the maximum principle for continuous functions, Laplacian comparison theorems, constructions of rays, lines and Busemann functions. For more details on the subject see [11. In this paper we give an alternative proof and show that the reduction in holonomy can be obtained by just using the analytic set-up for Fredholm properties of an elliptic operator on noncompact manifolds. This analytic set-up was developed by Lockhart and McOwen, 8 and by Melrose, 9], 10.

\section{AsYmptoticALLY CYLINDRICAL MANIFOLDS}

In this section we first introduce the definitions of cylindrical and asymptotically cylindrical Riemannian manifolds.

Definition 2.1. An $n$-dimensional Riemannian manifold $\left(M_{0}, g_{0}\right)$ is called cylindrical if $M_{0}=X \times \mathbb{R}$ and $g_{0}$ is compatible with this product structure, that is,

$$
g_{0}=g_{X}+\mathrm{d} t^{2},
$$

where $X$ is a compact, connected $(n-1)$-manifold with Riemannian metric $g_{X}$.

Definition 2.2. A connected, complete $n$-manifold $(M, g)$ with $l$ cylindrical ends is called asymptotically cylindrical with decay rate $\beta=\left(\beta_{1}, \ldots, \beta_{1}\right) \in \mathbb{R}^{l}, \beta_{j}<0$ for $1 \leq j \leq l$, if there exist cylindrical $n$-manifolds $\left(M_{0 i}, g_{0 i}\right)$ with $M_{0 i}=X_{i} \times \mathbb{R}$ for connected $X_{i}$, a compact subset $K \subset M$, a real number $R$, and diffeomorphisms $\Psi_{i}: X_{i} \times(R, \infty) \rightarrow M \backslash K$, such that the pull-back metric $\left(\Psi_{i}\right)^{*}(g)$ satisfies $\left|\nabla_{0 i}^{k}\left(\left(\Psi_{i}\right)^{*}(g)-g_{0 i}\right)\right|=O\left(e^{\beta_{i} t}\right)$ on $X_{i} \times(R, \infty)$ for all $k \geqslant 0$, where $\nabla_{0 i}$ is the Levi-Civita connection of the cylindrical metric $g_{0 i}$ on $M_{0 i}=X_{i} \times \mathbb{R}$.

Remark 2.3. For simplicity, we will assume that the decay rates are all equal for each cylindrical end, that is, $\beta_{j}=\beta_{k}, 1 \leq j, k \leq l$.

2.1. An example. If there are additional geometric structures on the cylindrical manifold $\left(M_{0}, g_{0}\right)=\left(X \times \mathbb{R}, g_{0}\right)$, then they should also be compatible with this product structure. In addition to the metric $g$, the geometric structures on the asymptotically cylindrical manifold $(M, g)$ should converge to order $O\left(e^{\beta t}\right)$ to the cylindrical geometric structures on $\left(M_{0}, g_{0}\right)$, with all of their derivatives.

One example where we have such additional structures on $(M, g)$ is an asymptotically cylindrical $G_{2}$-manifold. A 7-dimensional Riemannian manifold $(M, g)$ is called a $G_{2}$-manifold if the holonomy group of its Levi-Civita connection of $g$ lies inside of $G_{2} \subset S O(7)$. A $G_{2}$-manifold is equipped with a vector cross product $\times$ on its tangent bundle and a harmonic (calibration) 3 -form $\varphi \in \Omega^{3}(M)$ such that

$$
\varphi(u, v, w)=g(u \times v, w)
$$

for tangent vectors $u, v, w \in T(M)$. For more information on $G_{2}$-manifolds see [3].

Next, we define an asymptotically cylindrical $G_{2}$-manifold with one end.

Let $\left(M_{0}, \varphi_{0}, g_{0}\right)$ be a $G_{2}$-manifold with calibration 3-form $\varphi_{0}$ and the Riemannian metric $g_{0}$. In this case Definitions 2.1 and 2.2 take the following form, as in [4]. 
Definition 2.4. A $G_{2}$-manifold $\left(M_{0}, \varphi_{0}, g_{0}\right)$ is called cylindrical if $M_{0}=X \times \mathbb{R}$ and $\left(\varphi_{0}, g_{0}\right)$ is compatible with this product structure, that is,

$$
\varphi_{0}=\operatorname{Re} \Omega+\omega \wedge \mathrm{d} t \quad \text { and } \quad g_{0}=g_{X}+\mathrm{d} t^{2},
$$

where $X$ is a compact, connected Calabi-Yau 3-fold with Kähler form $\omega$, Riemannian metric $g_{X}$ and holomorphic $(3,0)$-form $\Omega$.

Definition 2.5. A connected, complete $G_{2}$-manifold $(M, \varphi, g)$ is called asymptotically cylindrical with decay rate $\beta, \beta<0$, if there exists a cylindrical $G_{2}$-manifold $\left(M_{0}, \varphi_{0}, g_{0}\right)$ with $M_{0}=X \times \mathbb{R}$ as above, a compact subset $K \subset M$, a real number $R$, and a diffeomorphism $\Psi: X \times(R, \infty) \rightarrow M \backslash K$ such that $\Psi^{*}(\varphi)=\varphi_{0}+\mathrm{d} \xi$ for some smooth 2 -form $\xi$ on $X \times(R, \infty)$ with $\left|\nabla_{0}^{k} \xi\right|=O\left(e^{\beta t}\right)$ on $X \times(R, \infty)$ for all $k \geqslant 0$, where $\nabla_{0}$ is the Levi-Civita connection of the cylindrical metric $g_{0}$.

Using similar relations between the geometric structures we can define cylindrical and asymptotically cylindrical Calabi-Yau manifolds. The details of these definitions and the applications will appear in a forthcoming paper, 14.

2.2. The Laplacian operator on asymptotically cylindrical manifolds. In this section we prove Proposition 2.11 and study the properties of the cokernel of the asymptotically cylindrical Laplacian operator. This proposition plays an important role in Section 3 in the proof of Theorem 1.1.

First we recall the basic facts about the properties of the Laplacian operator and its (co)kernel on asymptotically cylindrical manifolds with decay rate $\beta<0$. More details can be found in Lockhart and McOwen [7, [8. Also one can find similar results with a different analytical approach in Melrose, 9], [10].

Definition 2.6. Let $(M, g)$ be an asymptotically cylindrical Riemannian manifold asymptotic to $X \times \mathbb{R}$ with decay rate $\beta$. Let $\Delta_{0}: C^{\infty}(X \times \mathbb{R}) \rightarrow C^{\infty}(X \times \mathbb{R})$ be the cylindrical Laplacian operator defined on the Riemannian cylinder which is the asymptotic model for the ends of $M$ and invariant under translations in $\mathbb{R}$. Also, let $\Delta: C^{\infty}(M) \rightarrow C^{\infty}(M)$ be the Laplacian operator on $M$. Suppose that $\Psi$ is defined as in Definition 2.2. Then since $g$ is an asymptotically cylindrical metric asymptotic to $g_{0}, \Delta$ is asymptotic to $\Delta_{0}$ and $\Psi_{*}(\Delta)=\Delta_{0}+O\left(e^{\beta t}\right)$ as $t \rightarrow \infty$ for $\beta<0$, in the sense that their coefficients (in local coordinates on $X$ and $\mathbb{R}$ ) are exponentially close. We call $\Delta$ the asymptotically cylindrical Laplacian operator.

Choose a smooth function $\rho: M \rightarrow \mathbb{R}$ such that $\Psi^{*}(\rho) \equiv t$ on $X \times(R, \infty)$. This prescribes $\rho$ on $M \backslash K$, so we only have to extend $\rho$ over the compact set $K$. For $p \geq 1, k \geq 0$ and $\alpha \in \mathbb{R}$ we define the weighted Sobolev space $L_{k, \alpha}^{p}(M)$ to be the set of functions $f$ on $M$ that are locally integrable and $k$ times weakly differentiable and for which the norm

$$
\|f\|_{L_{k, \alpha}^{p}}=\left(\sum_{j=0}^{k} \int_{M} e^{-\alpha \rho}\left|\nabla^{j} f\right|^{p} \mathrm{~d} V\right)^{1 / p}
$$

is finite. Then $L_{k, \alpha}^{p}(M)$ is a Banach space. Since $\rho$ is uniquely determined except on the compact set $K$, different choices of $\rho$ give the same space $L_{k, \alpha}^{p}(M)$, with equivalent norms. 
Let $L_{k, \alpha}^{p}(M)$ be the weighted Sobolev spaces for some $\alpha$ such that $\beta<\alpha<0$. Then one can show that $\Delta$ extends to bounded linear operators

$$
\Delta_{k, \alpha}^{p}: L_{k, \alpha}^{p}(M) \longrightarrow L_{k-2, \alpha}^{p}(M)
$$

for all $p>1, k \geqslant 2$ and $\alpha \in \mathbb{R}$.

In Definition 2.7 we define a set $\mathcal{D}_{\Delta_{0}} \subset \mathbb{R}$, which determines the value of $\alpha$ so that an asymptotically cylindrical operator is Fredholm.

Definition 2.7. Let $\Delta$ and $\Delta_{0}$ be the Laplacian operators on $M$ and $X \times \mathbb{R}$. Extend $\Delta_{0}$ to the complexifications $\Delta_{0}: C^{\infty}(X \times \mathbb{R}) \otimes \mathbb{C} \rightarrow C^{\infty}(X \times \mathbb{R}) \otimes \mathbb{C}$. Then $\mathcal{D}_{\Delta_{0}}$ is the set of $\alpha \in \mathbb{R}$ such that for some $\delta \in \mathbb{R}$ there exists a nonzero section $s \in C^{\infty}(X \times \mathbb{R}) \otimes \mathbb{C}$ invariant under translations in $\mathbb{R}$ such that $\Delta_{0}\left(e^{(\alpha+i \delta) t} s\right)=0$.

Lockhart and McOwen prove [8, Th. 1.1] that (2) is Fredholm if and only if $\alpha$ does not lie in a discrete set $\mathcal{D}_{\Delta_{0}} \subset \mathbb{R}$.

Theorem 2.8. Let $(M, g)$ be an asymptotically cylindrical Riemannian manifold asymptotic to $\left(X \times \mathbb{R}, g_{0}\right)$, and let $\Delta: C^{\infty}(M) \rightarrow C^{\infty}(M)$ be the asymptotically cylindrical Laplacian operator on $M$ between functions on $M$, asymptotic to the cylindrical Laplacian operator $\Delta_{0}: C^{\infty}(X \times \mathbb{R}) \rightarrow C^{\infty}(X \times \mathbb{R})$ on $X \times \mathbb{R}$. Let $\mathcal{D}_{\Delta_{0}}$ be defined as above.

Then $\mathcal{D}_{\Delta_{0}}$ is a discrete subset of $\mathbb{R}$, and for $p>1, k \geqslant 2$ and $\alpha \in \mathbb{R}$, the extension $\Delta_{k, \alpha}^{p}: L_{k, \alpha}^{p}(M) \rightarrow L_{k-2, \alpha}^{p}(M)$ is Fredholm if and only if $\alpha \notin \mathcal{D}_{\Delta_{0}}$.

Also by an elliptic regularity result [7, Th. 3.7.2] and the weighted Sobolev Embedding Theorem [7, Th. 3.10], we get

Theorem 2.9. For $\alpha \notin \mathcal{D}_{\Delta_{0}}$ the kernel $\operatorname{Ker}\left(\Delta_{k, \alpha}^{p}\right)$ is independent of $p, k$, and is a finite-dimensional vector space of smooth functions on $M$.

Theorem 2.10. Let $\Delta^{*}$ denote the adjoint operator of $\Delta$. Then for all $\alpha \notin \mathcal{D}_{\Delta_{0}}$, $p, q>1$ with $\frac{1}{p}+\frac{1}{q}=1$ and $k, m \geqslant 2$, there is a natural isomorphism

$$
\operatorname{Coker}\left(\Delta_{k, \alpha}^{p}\right) \cong \operatorname{Ker}\left(\left(\Delta^{*}\right)_{m,-\alpha}^{q}\right)^{*}
$$

We now prove the following proposition. A related result with similar calculations can be found in Lockhart and McOwen, [8, Th. 7.4].

Proposition 2.11. Let $M$ be an orientable, asymptotically cylindrical Riemannian manifold with $l$ cylindrical ends $X_{1} \times(R, \infty), \ldots, X_{l} \times(R, \infty)$. Let $\Delta_{k, \alpha}^{p}$ be the asymptotically cylindrical Laplacian operator on $M$ for some small $\alpha, \alpha<0,[\alpha, 0) \cap$ $\mathcal{D}_{\Delta_{0}}=\emptyset$. Then

$$
\operatorname{dim}\left(\operatorname{Coker}\left(\Delta_{k, \alpha}^{p}\right)\right)=\operatorname{dim}\left(\operatorname{Ker}\left(\left(\Delta^{*}\right)_{m,-\alpha}^{q}\right)^{*}\right)=l .
$$

Proof. When $\alpha \notin \mathcal{D}_{\Delta_{0}}$ we see from (3) that

$$
\begin{aligned}
\operatorname{ind}\left(\Delta_{k, \alpha}^{p}\right) & =\operatorname{dim} \operatorname{Ker}\left(\Delta_{k, \alpha}^{p}\right)-\operatorname{dim} \operatorname{Ker}\left(\left(\Delta^{*}\right)_{m,-\alpha}^{q}\right), \\
\operatorname{ind}\left(\Delta_{m,-\alpha}^{q}\right) & =\operatorname{dim} \operatorname{Ker}\left(\Delta_{m,-\alpha}^{q}\right)-\operatorname{dim} \operatorname{Ker}\left(\left(\Delta^{*}\right)_{k, \alpha}^{p}\right) .
\end{aligned}
$$

Now since $\Delta$ is self adjoint, $\Delta=\Delta^{*}$, we have

$$
\operatorname{ind}\left(\Delta_{m,-\alpha}^{q}\right)=-\operatorname{ind}\left(\Delta_{k, \alpha}^{p}\right)=\operatorname{dim} \operatorname{Ker}\left(\Delta_{m,-\alpha}^{q}\right)-\operatorname{dim} \operatorname{Ker}\left(\Delta_{k, \alpha}^{p}\right) .
$$


Let $M$ be an asymptotically cylindrical Riemannian manifold with one cylindrical end $X \times(R, \infty)$. Lockhart and McOwen show [8, Th. 6.2] that for $\alpha, \delta \in \mathbb{R} \backslash \mathcal{D}_{\Delta_{0}}$ with $\alpha \leq \delta$ we have

$$
\operatorname{ind}\left(\Delta_{k, \delta}^{p}\right)-\operatorname{ind}\left(\Delta_{k, \alpha}^{p}\right)=\sum_{\epsilon \in \mathcal{D}_{\Delta_{0}}: \alpha<\epsilon<\delta} d(\epsilon),
$$

where $d(\epsilon) \geqslant 1$ is the dimension of the vector space of solutions $f \in C^{\infty}(X \times \mathbb{R}) \otimes \mathbb{C}$ of a prescribed form as in Definition 2.7 with $\Delta_{0} f=0$.

As $[\alpha,-\alpha] \cap \mathcal{D}_{\Delta_{0}}=\{0\}$, we can show that

$$
\operatorname{ind}\left(\Delta_{k,-\alpha}^{p}\right)-\operatorname{ind}\left(\Delta_{k, \alpha}^{p}\right)=2 b^{0}(X) .
$$

By (7), $\operatorname{ind}\left(\Delta_{m,-\alpha}^{q}\right)-\operatorname{ind}\left(\Delta_{k, \alpha}^{p}\right)$ is the dimension of the solution space of $\Delta_{0} f=0$ on $X \times \mathbb{R}$ for $f$ polynomial in $t \in \mathbb{R}$. By Hodge theory we deduce (8). By Theorems 2.9 and 2.10 we can conclude that the dimensions of kernel and cokernel are independent of $p, k$, and we can rewrite (8) as

$$
\operatorname{ind}\left(\Delta_{m,-\alpha}^{q}\right)-\operatorname{ind}\left(\Delta_{k, \alpha}^{p}\right)=2 b^{0}(X) .
$$

Now let $M$ be an asymptotically cylindrical Riemannian manifold with $l$ cylindrical ends $X_{1} \times(R, \infty), \ldots, X_{l} \times(R, \infty)$. In this case we get a contribution of 2 dimensions for each end, and equation (9) becomes

$$
\operatorname{ind}\left(\Delta_{m,-\alpha}^{q}\right)-\operatorname{ind}\left(\Delta_{k, \alpha}^{p}\right)=2 l .
$$

Now since we have from equation (6) that $\operatorname{ind}\left(\Delta_{m,-\alpha}^{q}\right)=-\operatorname{ind}\left(\Delta_{k, \alpha}^{p}\right)$, we get

$$
\operatorname{ind}\left(\Delta_{m,-\alpha}^{q}\right)=\operatorname{dim} \operatorname{Ker}\left(\Delta_{m,-\alpha}^{q}\right)-\operatorname{dim} \operatorname{Ker}\left(\Delta_{m, \alpha}^{q}\right)=l .
$$

But by maximum principle the harmonic functions which decay with rate $\alpha$, $\alpha<0$ cannot be nonzero functions, and one can easily see that $\operatorname{Ker}\left(\Delta_{m, \alpha}^{q}\right)=0$. This with equation (11) yields the result.

\section{Proof of Theorem 1.1}

In order to prove Theorem 1.1 we need to construct a closed and co-closed one form $\gamma=\mathrm{d} f$ for some harmonic function $f$ on $M$ and show that $\nabla \gamma=0$.

3.1. Construction of $f$ on $M$. We start by constructing a function $f$ which is harmonic on $M$ with $l$ cylindrical ends $X_{1} \times(R, \infty), \ldots, X_{l} \times(R, \infty)$. Let $f_{0}$ be a smooth function on $M$ of the form $f_{0}=C_{i} t_{i}+D_{i}$ on $X_{i} \times(R, \infty)$ for each $i=1, \ldots, l$. Here $t_{1}, \ldots, t_{l}$ are coordinates on cylindrical ends and $C_{i}, D_{i} \in \mathbb{R}$. The function $f_{0}$ smoothly interpolates between the cylindrical ends. The harmonic function $f$ on $M$ is of the form $f=f_{0}+f^{\prime}$ for some function $f^{\prime}$. Using elliptic estimates, [7. Th. 3.7.2] and embeddings of Sobolev spaces into Hölder spaces [1, Sec.2.7.], one can take $f^{\prime} \in L_{k, \alpha}^{p}(M)$ to be smooth, which decays like $O\left(e^{\alpha t}\right)$. Therefore $f=C_{i} t_{i}+D_{i}+O\left(e^{\alpha t_{i}}\right), \alpha<0$, on each end $X_{i} \times \mathbb{R}$.

In order to show the existence of the function $f$ such that $f=f_{0}+f^{\prime}$, we need to find a function $f^{\prime}$ that satisfies

$$
\Delta f^{\prime}=-\Delta f_{0} .
$$

This is equivalent to showing that $\Delta f_{0} \perp \operatorname{Coker}\left(\Delta_{k, \alpha}^{p}\right)$, and in Proposition 3.1 we prove this fact. Let $f_{0}=f_{0}^{\left(C_{i}, D_{i}\right)}$ be defined as above. 
Proposition 3.1. Let $M$ be an asymptotically cylindrical Riemannian manifold with l cylindrical ends $X_{1} \times(R, \infty), \ldots, X_{l} \times(R, \infty)$. Also for $h \in\left(\operatorname{Coker}\left(\Delta_{k, \alpha}^{p}\right)\right)^{*}$ let $\Phi$ be a map defined as

$$
\begin{aligned}
\Phi:\left(C_{i}, D_{i}\right) & \longrightarrow \operatorname{Coker}\left(\Delta_{k, \alpha}^{p}\right), \\
\Phi\left(C_{i}, D_{i}\right): h & \longmapsto\left\langle\Delta f_{0}^{\left(C_{i}, D_{i}\right)}, h\right\rangle_{L^{2}},
\end{aligned}
$$

where $\langle\cdot, \cdot\rangle_{L^{2}}$ is the $L^{2}$-inner product defined on the space of functions on $M$.

Then for any element $\left(C_{i}, D_{i}\right) \in \operatorname{Ker} \Phi$ the map $\operatorname{Ker} \Phi \longrightarrow\left(\operatorname{Coker}\left(\Delta_{k, \alpha}^{p}\right)\right)^{*}$ given by

$\left(C_{i}, D_{i}\right) \longrightarrow\left\langle f^{\left(C_{i}, D_{i}\right)}\right| \Delta f^{\left(C_{i}, D_{i}\right)}=0, f^{\left(C_{i}, D_{i}\right)}=C_{i} t_{i}+D_{i}+O\left(e^{\alpha t_{i}}\right)$ on $\left.X_{i} \times(R, \infty)\right\rangle$

is an isomorphism.

Proof. First note that $\Phi$ is linear in $\left(C_{i}, D_{i}\right)$, as we defined $f_{0}^{\left(C_{i}, D_{i}\right)}$ to be linear in $\left(C_{i}, D_{i}\right)$. Since there are $2 l$ parameters in $\left(C_{i}, D_{i}\right)$ and also $\operatorname{dim} \operatorname{Coker}\left(\Delta_{k, \alpha}^{p}\right)=l$ from Proposition 2.11, $\Phi$ is a linear map between $\mathbb{R}^{2 l} \rightarrow \mathbb{R}^{l}$ which guarantees that $\operatorname{dim} \operatorname{Ker} \Phi \geq l$. But for each $\left(C_{i}, D_{i}\right) \in \operatorname{Ker} \Phi$, if $\Delta f_{0}^{\left(C_{i}, D_{i}\right)} \perp \operatorname{Coker}\left(\Delta_{k, \alpha}^{p}\right)$, then $\left(f^{\prime}\right)^{\left(C_{i}, D_{i}\right)} \in L_{k, \alpha}^{p}(M)$ exists, and this implies that there exists an $f^{\left(C_{i}, D_{i}\right)}$ such that $\Delta f^{\left(C_{i}, D_{i}\right)}=0$ and $f^{\left(C_{i}, D_{i}\right)}=C_{i} t_{i}+D_{i}+O\left(e^{\alpha t_{i}}\right)$ on the $i$ th end.

Therefore we have an injective linear map

$\operatorname{Ker} \Phi \longrightarrow\left\langle f^{\left(C_{i}, D_{i}\right)} \mid \Delta f^{\left(C_{i}, D_{i}\right)}=0, f^{\left(C_{i}, D_{i}\right)}=C_{i} t_{i}+D_{i}+O\left(e^{\alpha t_{i}}\right)\right\rangle \subset\left(\operatorname{Coker}\left(\Delta_{k, \alpha}^{p}\right)\right)^{*}$, and hence we get an injective linear map

$$
\begin{aligned}
\operatorname{Ker} \Phi & \longrightarrow\left(\operatorname{Coker}\left(\Delta_{k, \alpha}^{p}\right)\right)^{*} \\
\left(C_{i}, D_{i}\right) & \longmapsto f^{\left(C_{i}, D_{i}\right)}=f_{0}^{\left(C_{i}, D_{i}\right)}+\left(f^{\prime}\right)^{\left(C_{i}, D_{i}\right)} .
\end{aligned}
$$

This implies that $\operatorname{dim} \operatorname{Ker} \Phi \leq \operatorname{dim}\left(\operatorname{Coker}\left(\Delta_{k, \alpha}^{p}\right)\right)^{*}=l$, and hence $\operatorname{dim} \operatorname{Ker} \Phi=l$. Therefore $\operatorname{Ker} \Phi \rightarrow\left(\operatorname{Coker}\left(\Delta_{k, \alpha}^{p}\right)\right)^{*}$ is an isomorphism as an injective linear map between spaces of dimension $l$, and this completes the proof.

3.2. Existence of constant harmonic 1-form $\gamma=d f$ on $M$. After constructing the harmonic function $f$, one can easily obtain a closed and coclosed 1-form $\gamma=d f$ on $M$. We now show that $\gamma=d f$ is constant for Ricci-flat manifolds. Let $g$ be the Riemannian metric on $M$. In the index notation the Weitzenböck formula for a 1 -form $\xi$ is

$$
\left(\mathrm{dd}^{*}+\mathrm{d}^{*} \mathrm{~d}\right) \xi_{a}=\nabla^{*} \nabla \xi_{a}+R_{a b} g^{b c} \xi_{c}
$$

where $R_{a b}$ is the Ricci curvature of $g$. Suppose that $R_{a b}=0$. Then we have

$$
\left(\mathrm{dd}^{*}+\mathrm{d}^{*} \mathrm{~d}\right) \xi=\nabla^{*} \nabla \xi
$$

on 1-forms on a Ricci-flat manifold $M$.

Proposition 3.2. Let $M$ be an asymptotically cylindrical Riemannian manifold with Ricci-flat metric $g$. Let $\gamma=d f$ be a harmonic 1-form defined as above. Then $\gamma=d f$ is constant, that is, $\nabla \gamma=0$. 
Proof. Let $K_{R}=M \backslash\left(\left(X_{1} \times(R, \infty) \cup \ldots \cup\left(X_{l} \times(R, \infty)\right)\right), R \gg 0\right.$. Then $\partial K_{R}=$ $\left(X_{1} \times\{R\}\right) \cup \ldots \cup\left(X_{l} \times\{R\}\right)$. Since $\gamma$ is harmonic we have $\left(\mathrm{dd}^{*}+\mathrm{d}^{*} \mathrm{~d}\right) \gamma=0$, and by equation (16) we get

$$
\int_{K_{R}} \gamma\left(\left(\mathrm{dd}^{*}+\mathrm{d}^{*} \mathrm{~d}\right) \gamma\right)=\int_{K_{R}} \gamma\left(\nabla^{*} \nabla \gamma\right)=0 .
$$

By Stoke's Theorem,

$$
\int_{K_{R}}|\nabla \gamma|^{2}=\int_{X_{1} \times\{R\}} \gamma \cdot\left(\nabla \gamma \cdot \eta_{1}\right)+\ldots+\int_{X_{l} \times\{R\}} \gamma \cdot\left(\nabla \gamma \cdot \eta_{l}\right)
$$

for normal vectors $\eta_{1}, \ldots, \eta_{l}$. The asymptotic behaviour of $\gamma=d f$ guarantees that the right-hand side of equation (18) goes to zero as $R \rightarrow \infty$ and yields the result.

We now complete the proof of Theorem 1.1. Let $(M, g)$ be an asymptotically cylindrical Ricci-flat manifold with $l$ ends. Then $(M, g)$ is asymptotic to the cylinders $X_{1} \times(R, \infty), \ldots, X_{l} \times(R, \infty)$, for $R \gg 0$. Proposition 3.1 guarantees the existence of a harmonic function $f$ on $M$.

Note that if $l=1$, by Proposition 2.11, $\operatorname{dim}\left(\operatorname{Coker}\left(\Delta_{k, \alpha}^{p}\right)\right)=1$ and $f$ should be constant, so $d f=0$. If $l \geqslant 2$, then one can choose $f$ not constant and construct a nonzero, closed and coclosed 1-form $\gamma=d f$. Since $(M, g)$ is Ricci-flat, by Proposition $3.2 \gamma$ is constant. Thus the results [3, Sec. 3.2] show that the metric $g$ on $M$ is locally reducible. Rescale $f$ if necessary so that $|\gamma| \equiv 1$.

Also note that by Proposition 3.2 $d f$ is covariant constant and so $|d f|$ is constant. Therefore $\gamma=d f$ never vanishes on $M$, and $f$ can be viewed as a Morse function with no critical points. Define $X$ to be the level set $f^{-1}(0)$. This is a nonsingular submanifold of $M$ as $d f \neq 0$ everywhere, and is compact as $f \rightarrow \pm \infty$ at the ends of $M$. This implies that $M$ is at least a topological product $M=X \times \mathbb{R}$.

We now construct a diffeomorphism $X \times \mathbb{R} \rightarrow M$. For $t \in \mathbb{R}$ define $v_{t}: M \rightarrow M$ to be the gradient flow of $f$ for time $t$. As $M$ is complete and $|d f| \equiv 1$, this is a well-defined diffeomorphism, which induces a diffeomorphism between $X$ and the level set $f^{-1}(t)$. Define $\Upsilon: X \times \mathbb{R} \rightarrow M$ by $\Upsilon(x, t)=v_{t}(x)$. This is a bijection, as for each $t, x \mapsto \Upsilon(x, t)=v_{t}(x)$ is a bijection between $X$ and $f^{-1}(t)$, and it is easy to see $\Upsilon$ is a diffeomorphism.

As $|d f| \equiv 1$, the metric $\Upsilon^{*}(g)$ is of the form $g(x, t)+d t^{2}$, where $g(x, t)$ is a 1parameter family of metrics on $X$ depending on $t \in \mathbb{R}$. Furthermore, since $\Upsilon^{*}(g)$ is locally reducible and $d t$ is constant, we see that $g(x, t)$ is locally and hence globally independent of $t$. Thus $M$ is a Riemannian product $X \times \mathbb{R}$. Since $M$ is connected $X$ is connected, so $M$ has exactly two ends, so $l=2$, and it is a cylinder.

Remark 3.3. If $(M, g)$ is an asymptotically cylindrical $G_{2}$ manifold with two cylindrical ends, then $\operatorname{Hol}(g)$ preserves $\gamma$ and there is a reduction in the holonomy group, and hence $\operatorname{Hol}(g) \subset S U(3)$. This implies that $M=X \times \mathbb{R}$ where $X$ is a Calabi-Yau 3 -fold, and hence $M$ is a cylinder.

\section{ACKNOWLEDGEMENTS}

The author is grateful to Dominic Joyce for his help and encouragement during this project and to the AWM-NSF for their grant support. Special thanks to the referee for many valuable comments. 


\section{REFERENCES}

[1] Aubin, T., Nonlinear Analysis on Manifolds. Monge-Ampère Equations, Springer-Verlag (1982). MR0681859 (85j:58002)

[2] Cheeger, J. and Gromoll, D., The splitting theorem for manifolds of nonnegative Ricci curvature, J. Differential Geometry 6 (1971/72), 119-128. MR0303460 (46:2597)

[3] Joyce, D.D., Compact Manifolds with Special Holonomy, OUP, Oxford, 2000. MR1787733 (2001k:53093)

[4] Joyce, D.D. and Salur, S., Deformations of Asymptotically Cylindrical Coassociative Submanifolds with Fixed Boundary, Geom. Topol. 9 (2005), 1115-1146 (electronic). MR2140999

[5] Kovalev, A.G., Twisted connected sums and special Riemannian holonomy, J. Reine Angew. Math. 565 (2003), 125-160. MR.2024648 (2004m:53088)

[6] Leung, C.L., Topological Quantum Field Theory for Calabi-Yau threefolds and $G_{2}$ manifolds, Adv. Theor. Math. Phys. 6 (2002), no. 3, 575-591. MR1957671 (2004m:53095)

[7] Lockhart, R.B., Fredholm, Hodge and Liouville Theorems on noncompact manifolds, Trans. A.M.S. 301 (1987), 1-35. MR0879560 (88g:58190)

[8] Lockhart, R.B. and McOwen, R.C., Elliptic differential operators on noncompact manifolds, Ann. Scuola Norm. Sup. Pisa, Classe di Scienze 12 (1987), 409-447. MR0837256 (87k:58266)

[9] Melrose, R., The Atiyah-Patodi-Singer Index Theorem, A.K. Peters, Wellesley, MA, 1994. MR.1348401 (96g:58180)

[10] Melrose, R., Spectral and Scattering Theory for the Laplacian on Asymptotically Euclidean Spaces, in Ikawa M., editor, Spectral and Scattering Theory, Lecture notes in Pure and Applied Mathematics, vol. 161, Marcel Dekker, Inc., 1994. MR1291640 (95k:58168)

[11] Petersen, P., Riemannian Geometry, Graduate Texts in Mathematics 171, Springer (1998). MR.1480173 (98m:53001)

[12] Tian, G. and Yau, S.T., Complete Kähler manifolds with zero Ricci curvature I, J. Amer. Math. Soc. 3 (1990), 579-609. MR1040196 (91a:53096)

[13] Tian, G. and Yau, S.T., Complete Kähler manifolds with zero Ricci curvature II, Invent. Math. 106 (1991), 27-60. MR1123371 (92j:32028)

[14] Salur, S., Special Lagrangian Submanifolds with Cylindrical Ends, in preparation, 2006.

Department of Mathematics, Northwestern University, Evanston, Illiinois 60208

E-mail address: salur@math.northwestern.edu 